\title{
BMJ Open Default options in advance directives: study protocol for a randomised clinical trial
}

\author{
Nicole B Gabler, ${ }^{1,2}$ Elizabeth Cooney, ${ }^{1,2}$ Dylan S Small, ${ }^{3}$ Andrea B Troxel, ${ }^{2}$ \\ Robert M Arnold, ${ }^{4}$ Douglas B White, ${ }^{4}$ Derek C Angus, ${ }^{4}$ George Loewenstein, ${ }^{5}$ \\ Kevin G Volpp, ${ }^{6,7,8,1,2}$ Cindy L Bryce, ${ }^{4}$ Scott D Halpern ${ }^{6,7,8,1,2}$
}

To cite: Gabler NB, Cooney E, Small DS, et al. Default options in advance directives: study protocol for a randomised clinical trial. BMJ Open 2016;6:e010628. doi:10.1136/bmjopen-2015010628

- Prepublication history and additional material is available. To view please visit the journal (http://dx.doi.org/ 10.1136/bmjopen-2015010628).

Received 23 November 2015 Revised 29 March 2016 Accepted 26 April 2016

CrossMark

For numbered affiliations see end of article.

\section{Correspondence to} Dr Scott D Halpern; shalpern@exchange.upenn. edu

\section{ABSTRACT}

Introduction: Although most seriously ill Americans wish to avoid burdensome and aggressive care at the end of life, such care is often provided unless patients or family members specifically request otherwise. Advance directives (ADs) were created to provide opportunities to set limits on aggressive care near life's end. This study tests the hypothesis that redesigning ADs such that comfort-oriented care is provided as the default, rather than requiring patients to actively choose it, will promote better patient-centred outcomes.

Methods and analysis: This multicentre trial randomises seriously ill adults to receive 1 of 3 different ADs: (1) a traditional AD that requires patients to actively choose their goals of care or preferences for specific interventions (eg, feeding tube insertion) or otherwise have their care guided by their surrogates and the prevailing societal default toward aggressive care; (2) an $A D$ that defaults to life-extending care and receipt of life-sustaining interventions, enabling patients to opt out from such care; or (3) an AD that defaults to comfort care, enabling patients to opt into life-extending care. We seek to enrol 270 patients who return complete, legally valid $A D s$ so as to generate sufficient power to detect differences in the primary outcome of hospital-free days (days alive and not in an acute care facility). Secondary outcomes include hospital and intensive care unit admissions, costs of care, hospice usage, decision conflict and satisfaction, quality of life, concordance of preferences with care received and bereavement outcomes for surrogates of patients who die.

Ethics and dissemination: This study has been approved by the Institutional Review Boards at all trial centres, and is guided by a data safety and monitoring board and an ethics advisory board. Study results will be disseminated using methods that describe the results in ways that key stakeholders can best understand and implement.

Trial registration number: NCT02017548; Pre-results.

\section{BACKGROUND}

Most seriously ill Americans wish to die at home and to avoid aggressive and burdensome care near the end of life. ${ }^{12}$ However,

\section{Strengths and limitations of this study}

- Uses a unique study design and employs principles of behavioural economics to assess the impact of default options in advance directives.

- Leverages changes in treatment goals and preferences to assess the impact on clinical, economic and patient-reported and surrogate-reported outcomes.

- Many randomised patients will not complete their assigned advance directives.

roughly one half of deaths in the USA occur in the hospital, ${ }^{3} 20 \%$ of Americans die in or shortly following a stay in the intensive care unit (ICU); $;^{4}$ one in three elderly patients undergo an inpatient surgical procedure during their last year of life $;^{5}$ one in two elderly Americans visits an emergency department in their last month of life $;^{6}$ and more than one-quarter of Medicare dollars are spent on patients in their final year. ${ }^{4} 7$ Aggressive treatments at the end of life are also associated with reduced quality ${ }^{8} 9$ and quantity of life,,$^{10-13}$ and may produce longlasting pathological bereavement among family members making decisions about their loved ones' end-of-life care. ${ }^{8} 9$ 14-17

Written advance directives (ADs) have the potential to reduce the discrepancy between the care a patient desires and the care a patient receives. Currently, critical healthcare decisions must be made for $43 \%$ of older Americans near the end of life, but $70 \%$ of these patients are unable to participate in making these decisions ${ }^{18}$ and must have decisions made for them. ADs, which include living wills and durable power of attorney designation, can help improve the quality of advance care planning. Observational studies show that elderly patients who complete ADs are more likely to die outside of a hospital, receive less costly care and receive care 
consistent with their preferences. ${ }^{18-21}$ However, ADs have known shortcomings, ${ }^{22-26}$ and finding a scalable solution for a diverse patient population is challenging.

The use of default options has been shown to have large effects in a variety of areas, including the use of opt out versus opt-in framing to increase organ donation and vaccination rates. ${ }^{27-32}$ Defaults are considered a powerful approach to help overcome a variety of problems in healthcare without limiting choice. ${ }^{33-36}$ In a pilot randomised clinical trial (RCT), ${ }^{37}$ we showed that default options in ADs may influence patients' choices of care, while preserving patients' satisfaction with the decision-making process, and that patients rarely changed their plan of care after being alerted to the intervention and their responses. However, before advocating default options in ADs for broader clinical use, it is necessary to determine whether changing such choices leads to improved patient-centred and familycentred outcomes over the long term in a larger, more diverse population of seriously ill patients. The current association of aggressive treatments at the end of life with poor patient-reported and surrogate-reported outcomes suggests that aligning the care patients desire with the care they receive, such that aggressive care is reduced at the end of life, will improve these outcomes.

\section{METHODS/DESIGN}

This study is an RCT examining whether structuring ADs to request comfort-oriented care or life-extending care by default influences the number of days that patients are alive and living outside of an acute-care hospital, as well as several secondary outcomes.

\section{Study hypothesis}

The primary study hypothesis is that ADs with preselected comfort care measures, compared with those defaulting to life-extension or standard ADs, will produce an increase in hospital-free days (HFDs), a measure that represents the number of days alive and not in an acute care facility. Secondary hypotheses are: compared with standard ADs or ADs defaulting to lifeextension, ADs defaulting to comfort care will (1) produce no change in survival; (2) reduce hospital and ICU admissions; (3) increase hospice usage; (4) reduce costs of inpatient plus hospice care; (5) improve patients' quality of life; (6) improve patients' satisfaction with care; (7) improve surrogates' perceptions of the quality of dying and death and (8) decrease the incidence of symptoms of post-traumatic stress among surrogates following their loved ones' deaths.

\section{Study setting}

Recruitment for the trial is occurring at several clinics associated with the University of Pennsylvania Health System in Philadelphia, Pennsylvania, and the University of Pittsburgh Medical Center in Pittsburgh,
Pennsylvania. We plan to recruit 270 patients who complete ADs. Recruitment started in February 2014.

\section{Eligibility criteria}

English-speaking patients 18 years or older are being recruited from the thoracic, gynaecological, gastrointestinal, genitourinary, liver and breast oncology, pulmonary, nephrology, movement disorder and heart failure clinics across the two Pennsylvania health systems. In order to be included in our study, patients must have specific diagnostic criteria (table 1) documented in their electronic health records. Criteria were selected in consultation with providers in the clinics listed above. We asked providers to help develop criteria that would define cohorts of patients for whom predicted survival is $<2$ years (median survival $\leq 24$ months).

Patients eligible for transplant are excluded because transplant would alter disease prognosis. Patients are selected based on prognosis and not age, because patients' goals tend to correspond more closely with time horizons related to prognosis than with age. ${ }^{38} 39$ We are limiting our patient population to residents of Pennsylvania and New Jersey to facilitate follow-up in statewide databases. Patients with prior living wills are excluded because one's choices in completing a prior living will may influence selections in subsequent ADs. Cognitively impaired patients are excluded from the study as they will not have capacity to consent or to make the required healthcare decisions on their $\mathrm{AD}$ forms. Cognitive impairment is primarily determined by physicians prior to research staff approaching the patient. Owing to our intervention being embedded in the actual $\mathrm{AD}$ forms, we are excluding patients who are unable to read English.

\section{Participant screening}

Each week, trained study personnel screen electronic medical records to identify eligible patients scheduled for outpatient follow-up visits the following week. A patient's eligibility status is entered into the eligibility database along with ICD9 and ICD10 codes, staging information, provider name, clinic location and upcoming appointments. Once an eligible patient is identified, a research coordinator emails the patient's provider to (1) inform the provider that the patient is eligible for recruitment and (2) provide an opportunity for the provider to decline or defer any patient's enrolment by responding to the email. Patients who meet eligibility criteria but are new patients (ie, not follow-up visits) are tracked in the eligibility database so they may be rescreened at a later date. If eligible, they will be approached for consent. Patients are only eligible for inclusion once. Once they consent or decline, patients are not approached again if they meet eligibility criteria in a different clinic. 
Table 1 Inclusion and exclusion criteria

\begin{tabular}{|c|c|}
\hline Inclusion & Exclusion \\
\hline $\begin{array}{l}\text { Age } 18 \text { years or older } \\
\text { Speaks and reads English fluently } \\
\text { Has seen current physician at least once prior to current visit } \\
\text { Resident of PA or NJ } \\
\text { One or more of the following diagnoses: } \\
\text { A. Amyotrophic lateral sclerosis } \\
\text { B. Stage IIIB or IV non-small-cell lung cancer or cholangiocarcinoma } \\
\text { C. Stage IV colorectal, oespophageal, gastric, pancreatic, prostate, uterine, } \\
\text { cervical, ovarian or urothelial cancer; paraganglioma or } \\
\text { pheochromocytoma } \\
\text { D. Stage C or D hepatocellular carcinoma } \\
\text { E. Stage IV renal cell carcinoma } \\
\text { F. Stage IV or V chronic kidney disease } \\
\text { G. Mesothelioma or any malignancy metastatic to the pleura } \\
\text { H. Other insurable interstitial lung diseases with at least severe restriction on } \\
\text { most recent pulmonary function tests or eligible for long-term oxygen } \\
\text { therapy } \\
\text { I. Chronic obstructive pulmonary disease with at least severe airflow } \\
\text { obstruction on most recent spirometry or eligible for long-term oxygen } \\
\text { therapy } \\
\text { J. Congestive heart failure with NYHA Class IV status or Class III plus } 1 \\
\text { heart failure-related hospitalisation in the past } 12 \text { months or ACC stage D } \\
\text { or C classification with } 1 \text { heart failure hospitalisation in the past } 12 \text { months } \\
\text { K. Stage IV breast cancer except patients whose only metastases are to the } \\
\text { bones or who are receiving endocrine therapy without receiving } \\
\text { concomitant traditional chemotherapy }\end{array}$ & $\begin{array}{l}\text { Currently listed for or being considered } \\
\text { for, solid organ transplant } \\
\text { - Previously signed advance directive or } \\
\text { living will } \\
\text { - Cognitive impairment }\end{array}$ \\
\hline
\end{tabular}

\section{Recruitment and retention}

Patients who screen eligible are approached by a research coordinator during routine clinic visits. The research coordinators seek patients' consent to participate in a study about healthcare decision-making. The consent forms (see online supplementary appendix A) contain Health Insurance Portability and Accountability Act statements of authorisation of release of medical records, and include clear explanations that they are being asked to complete an $\mathrm{AD}$, participate in several follow-up interviews and permit the research team to follow their health outcomes. Patients are also told that different types of ADs are assigned by chance, but that patients in all groups may select or decline any intervention or treatment goal, and may revise their choices at any time. All patients are encouraged to involve their family members and/or physicians in completing their ADs. All consenting patients are provided with: (1) their assigned $\mathrm{AD}$; (2) an informational brochure about ADs; (3) contact information for study personnel; (4) a copy of the Decisional Conflict Scale (DCS) and (5) instructions for returning the completed $\mathrm{AD}$ and DCS and a stamped envelope addressed to study staff. Demographics, including age, race, ethnicity, sex, religion, income, education, marital status and health insurance type are collected at the time of consent, as well as data about previous experience with life-sustaining therapies and critical care medicine. If a participant does not return an $\mathrm{AD}$ within 10 days, study personnel call every
10 days for 1 month, and again at 2 months and at 3 months, to encourage $\mathrm{AD}$, return or meet with the participant in person at his or her next clinic appointment. This specific contact frequency was guided by desire to limit missing data for important patient-centred outcomes.

Finally, we seek consent from patients' surrogates by phone or during clinic visits. Recruited surrogates are those identified in patients' ADs or if none, according to states' legal hierarchies. We tell surrogates that their roles are to (1) be a point of contact in the event that we are unable to reach the patient for follow-up; (2) participate in an interview related to surrogate outcomes and (3) complete the nine-item Healthcare System Distrust Scale. ${ }^{40}$ Patients who wish to participate, but lack a surrogate or do not want their surrogate to be contacted, are still eligible. Surrogates are not approached for consent if a patient has not completed an $\mathrm{AD}$.

Patients who do not wish to complete an $\mathrm{AD}$ and decline consent are asked to sign a limited consent form providing authorisation to access long-term health outcomes via electronic medical records and statewide databases, along with providing basic demographic information (age, race, ethnicity and gender). This information is essential to complete the proposed complier average treatment effect (or instrumental variable (IV)) analysis. No further contact with these patients is made.

Individuals will be contacted for participation in follow-up interviews 2, 6 and 12 months after $\mathrm{AD}$ 
completion. In order to maximise participation in follow-up interviews, participants are asked if they would prefer to be contacted by phone or email. For participants who wish to be contacted by phone, phone interviews will be conducted by a research assistant who is blinded to the participant's study arm and using a standardised script. Participants who prefer email contact will be sent a link to complete surveys online using the REDCap electronic data capture tool. ${ }^{41}$ This survey will include information and questions that are identical to the phone surveys. Surrogates will be interviewed if patients are unable to participate due to illness.

All consenting patients are compensated US $\$ 20$ for each follow-up interview. Surrogates are given US\$20 at the time of consent to encourage them to report patient deaths and to complete follow-up interviews with study staff.

\section{Randomisation and allocation concealment}

Consenting participants are randomised individually with a $33.3 \%$ probability to each trial arm (comfort-oriented defaults, life-extension defaults or standard $\mathrm{AD}$ ) using electronic randomisation managed by the Data Management Unit (DMU) at the University of Pennsylvania (see figure 1). Randomisation is stratified by centre using variable block sizes of three, six and nine patients to promote patient balance within the centre. Research coordinators remain blinded to patients' AD group until after consent is provided.

\section{Intervention}

The three $\mathrm{AD}$ forms used in this study are versions modified slightly from the professionally endorsed AD published by the Allegheny County Medical Society (see
Figure 1 Study scheme. AD, advance directives; QOL, quality of life; RC, research coordinator.

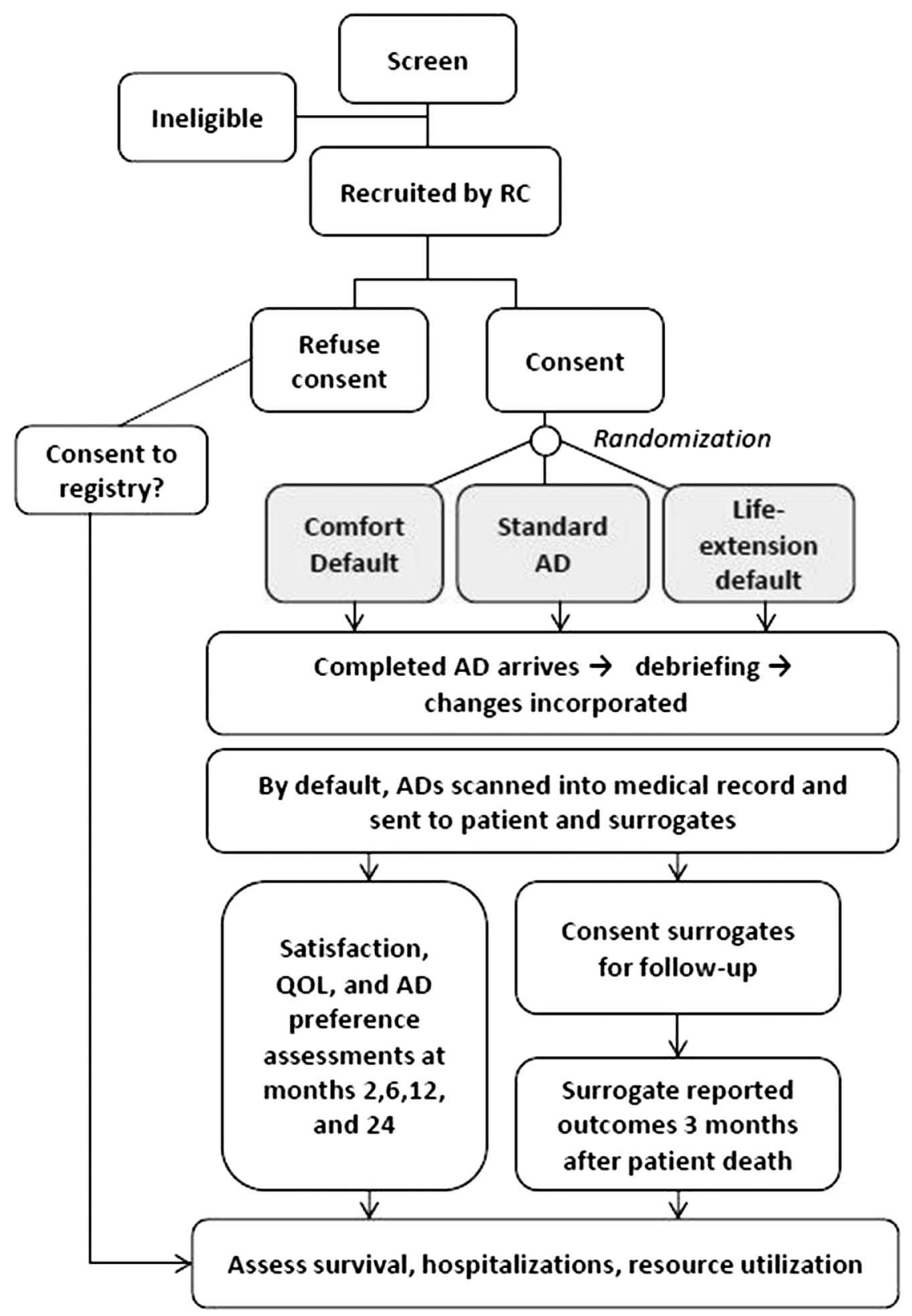


online supplementary appendix B).$^{42}$ Patients are asked to select between an overall plan of care focused on extending life or on relieving pain and suffering if these two goals are to come into conflict. The language used to describe these overall goals is taken directly from the SUPPORT trial. ${ }^{43}$ Additionally, patients are asked to choose whether or not they desire four specific lifesustaining interventions if they were to become sick and unable to make decision for themselves. These four interventions represent validated markers of end-of-life intensity $^{44}$ and are: cardiopulmonary resuscitation (CPR), mechanical ventilation, dialysis and feeding tube insertion for artificial nutrition and hydration. Finally, patients are asked to indicate the type of care they would want following hospital discharge (comfort-oriented without return visits to the hospital or life-extension including return visits to the hospital). For all questions, patients may select that they do not wish to specify, deferring the decision-making to their surrogate.

In the standard $\mathrm{AD}$, patients are asked to make active choices for each of these decisions. Patients not selecting a choice defer that decision to their surrogate. The life-extension and comfort default ADs are identical except that in these, the life-extension (or comfort-oriented) plan of care is preselected, as are choices to receive (or not) the four life-sustaining interventions, and the decision to continue (or forgo) intensive care following hospital discharge including future hospitalisations. Patients are clearly instructed that other preferences can be chosen by crossing out the preselected options and choosing alternatives or choosing that they do not wish to specify. The order of responses (comfort care vs life extension) is randomised for those patients assigned to the standard $\mathrm{AD}$ arm to mitigate any potential ordering effects. Patients randomised to the comfort care or life extension arms will see the life extension or comfort care default options first, respectively.

Patients in all groups are provided with an informational brochure about ADs that uses simple language, and research coordinators are trained to answer patients' questions about advance care planning and offer assistance to patients if necessary. Patients may also be referred to social work resources for additional assistance if desired.

\section{Debriefing}

Debriefing has long been used in 'deception research.' Although our study does not qualify as deceptive because we do not provide participants with untrue information, we believe debriefing remains an essential element of this RCT in which certain details are necessarily withheld during the consent process. As Wendler and colleagues note,${ }^{45}$ debriefing 'should be understood as a tool of moral accountability', and that 'debriefing makes amends by retrospectively providing the disclosure about the research that standardly should have been offered prospectively'. In our study, a thorough debriefing session will ensure that patients (1) understand their selections; (2) do not simply go with the default options because they failed to recognise that a choice was to be made or that a default was being used; (3) have multiple opportunities to withdraw their participation or data and (4) are actively engaged in the research and comfortable with the research process.

After receiving a patient's completed $\mathrm{AD}$ form, a research coordinator contacts the patient for a debriefing session. During the debriefing, the research coordinator uses an Institutional Review Board (IRB)-approved script (see online supplementary appendix C) that carefully explains the nature of the intervention, including the use of different default options in ADs. Patients are reminded that they received, by chance, one of three forms used in the study. The specific differences between all three versions are described, including explanations that choices were preselected, when appropriate. The research coordinator then reviews each $\mathrm{AD}$ choice with the patient and makes sure patients are satisfied with the selections before proceeding. Patients are specifically asked if they wish to change their selections on their $\mathrm{AD}$ forms, and are reminded that should their choices change in the future, they should contact the study team, their clinician or both. If the patient decides to make changes to his/her $\mathrm{AD}$, changes can be made immediately by the research coordinator and a new copy forwarded to the patient or the patient may choose to receive a blank copy of the $\mathrm{AD}$ and make new selections. Any changes will be incorporated, and the completed $\mathrm{AD}$ will be scanned into the patient's medical record and mailed to the patient, identified healthcare agents/ surrogates, and any other requested family members.

Once patients confirm that they are comfortable with their selections, the research coordinator informs patients that their ADs will be scanned into their medical records and sent to their home address as well as to their surrogates. Patients are given the opportunity to decline one or both of these efforts to promote $\mathrm{AD}$ accessibility. ADs will not be considered 'complete' until the debriefing session has occurred.

Debriefing primarily occurs over the phone, although a few patients who are unavailable via phone are debriefed in person. While we recognise that this procedure may not meet the needs of all patients, it is the best option in an RCT such as this and provides us with the ability to debrief all patients who complete and return an $\mathrm{AD}$ in a timely manner.

\section{Outcomes (primary and secondary)}

The primary outcome is HFDs (table 2). This metric represents the number of days alive and not in an acute care facility following the date of consent. We choose the date of consent as day 0 so that all enrolled participants, including those who do not return ADs, are eligible for intention-to-treat (ITT) analyses. However, to minimise immortal-time bias, we will also conduct a secondary analysis in which day 0 is defined as the date 
Table 2 Outcomes

\begin{tabular}{|c|c|}
\hline Outcome & Measurement \\
\hline Hospital-free days (primary outcome) & Number of days alive and not in an acute care facility \\
\hline Hospital and ICU admissions & Number of admissions analysed as count data \\
\hline Costs of care & $\begin{array}{l}\text { Combination of all costs of inpatient and outpatient } \\
\text { hospice, hospital stays and life-sustaining procedures }\end{array}$ \\
\hline Hospice usage & $\begin{array}{l}\text { Analysed as time from } \mathrm{AD} \text { completion to hospice } \\
\text { enrolment, and as duration of hospice usage prior to death }\end{array}$ \\
\hline $\begin{array}{l}\text { Choices to receive } 4 \text { potentially life-sustaining interventions } \\
\text { (CPR, mechanical ventilation, dialysis, feeding tube) }\end{array}$ & $\begin{array}{l}\text { Concordance of these choices with whether the } \\
\text { interventions were actually received }\end{array}$ \\
\hline $\begin{array}{l}\text { Choices regarding post-hospitalisation care (see online } \\
\text { supplementary appendix B for specific choices) }\end{array}$ & $\begin{array}{l}\text { Concordance of these choices with the care actually } \\
\text { received }\end{array}$ \\
\hline Decision conflict and satisfaction & $\begin{array}{l}\text { Decision Conflict Scale } \\
\text { CANHELP }\end{array}$ \\
\hline Quality of life & McGill quality of life \\
\hline Surrogates' perception of the quality of death and dying & Prigerson's quality of death \\
\hline Bereavement outcomes & Impact of Events Scale \\
\hline Healthcare system distrust & Healthcare System Distrust Scale \\
\hline
\end{tabular}

on which ADs are returned. Two additional variations on this metric will also be evaluated: (1) healthcare facility-free days, which represent the number of days alive where a patient is not in an acute care facility, a chronic care facility or a nursing home; and (2) HFDs within the first 6 months of follow-up.

The use of HFDs as our primary outcome reflects the desire to choose a measure that is patient-centred, readily measured and analysed, and reflects a patient's holistic state rather than a specific symptom. HFDs have many attractive properties: they are continuous, enhancing power; they can be analysed reliably and indeed flexibly, to account for different values patients may place on avoiding hospitalisation; and in nearly all cases, they are unidirectional, in the sense, that nearly all patients prefer longer lives to shorter ones, and to have more of those days spent outside a hospital than within. This does not automatically assume that all days spent in a hospital are without value. Indeed, the relief of acute symptoms via a short hospital stay may have great value to a patient, but such days will be a small percentage of total cohort days. And while these beneficial days may not be weighed differently in our model, they also would not reduce the number of remaining days a patient would have outside the hospital.

Secondary outcomes include several clinical, economic and patient-reported measures. Specifically, we capture patient deaths via medical records and, if necessary, we verify deaths in the Social Security Death Index, performing linkages via social security numbers, which are collected at the time of consent. We assess hospitalisations, ICU admissions, costs of inpatient care and usage of life-sustaining therapies by querying state-run databases that capture all admissions and inpatient procedures in Pennsylvania and New Jersey. These databases are also used to assess concordance between patients' choices to receive four potentially life-sustaining therapies (CPR, mechanical ventilation, dialysis and feeding tube), and whether those interventions are actually received. Data on hospice usage and costs are collected via data use agreements with organisations that provide care for $80 \%$ of patients at the University of Pennsylvania Health System and the University of Pittsburgh Medical Center.

In patient interviews conducted 2, 6 and 12 months following AD completion, we are assessing patients' satisfaction with their advance care planning, quality of life and desires to make any changes to their ADs. Satisfaction is measured with the Canadian Healthcare Evaluation Project (CANHELP) ${ }^{46} 47$ instrument's global satisfaction and end-of-life care question. This instrument asks patients 'how satisfied are you with your advance care planning overall-that is, the plans you have in place regarding your end-of-life care'. Responses range from 1 to 5 , with 1 indicating completely satisfied and 5 indicating not at all satisfied. Quality of life is measured using the McGill quality of life (MQoL), ${ }^{48} 49$ which can be completed by family members on behalf of patients who are unable to complete it themselves. The MQoL questionnaire has 16 questions querying both mental and physical symptoms experienced by the patient during the past 2 days, and asks patients to rate the severity of each. For example, the MQoL will prompt a patient with 'over the last two days, one troublesome symptom has been...', and the patient will self-identify a symptom. The patient will then be asked to rate on a 0 10 scale how problematic that symptom has been, with 0 indicating no problem and 10 indicating a tremendous problem. Patients will also be asked about their emotional well-being (eg, 'over the past 2 days, I have been depressed',) as well as support (eg, 'over the past 2 days, I have felt supported'), with responses ranging from not at all (0) to extremely or completely (10). Additionally, we are assessing decision conflict, using the validated 
DCS, ${ }^{50}$ to assess patients' certainty in making healthcare decisions. The DCS provides patients with 16 statements ('I know the benefits of each option', 'I am clear about which risks matter most to me'), and assesses agreement or disagreement with each statement on a 5-point scale ranging from strongly agree to strongly disagree. This measure is collected immediately following AD completion and is not assessed during follow-up.

We are assessing surrogates' perceptions of the quality of death and dying using Prigerson's quality of death measures. ${ }^{8} 951$ This three-question measure asks surrogates to rate, on a scale of $0-10$, the level of psychological distress or physical distress in the patient's last week of life, as well as the overall quality of the patient's death or last week of life. The Impact of Events Scale ${ }^{52}$ is used to assess the risk of post-traumatic stress disorder in surrogates of deceased patients. The surrogate is presented with 15 statements commonly made after stressful life events (eg, 'I tried to remove it from memory', and 'I had dreams about it'), and asked to respond with the frequency of how true the comments were for them during the last 7 days. Response options include not at all, rarely, sometimes and often. Finally, healthcare system distrust is measured among the surrogates using the Health System Distrust Scale. ${ }^{40}$ This scale asks surrogates to rate statements about the healthcare system on a 5-point Likert scale ranging from strongly disagree to strongly agree. Statements include items such as 'the health care system covers up its mistakes' and 'the health care system gives excellent medical care'.

\section{Data collection methods}

The DMU at the University of Pennsylvania manages all study data, and ensures secure multisite data integration, accurate merging of trial data with hospice and state claims databases, and processes scanning of report forms for demographics collection and all follow-up data to minimise errors. The database automatically tracks patients and notifies study personnel when follow-up interviews are due. Only authorised project personnel have access to the data, which is stored behind firewalls and not on stand-alone PCs or laptops. All study participants are assigned a study identification number, and any personally identifying information is removed from analytic data sets.

\section{Analytic plan}

First, we will use ITT analyses to assess the impact of group assignment on all outcomes. Second, we will use Complier Average Treatment Effect (CATE) analyses to assess the influence of the choices patients make in ADs on long-term patient-reported and family-reported outcomes, and to estimate the impact of completing an $\mathrm{AD}$ at all, regardless of the choices made. These methods are made feasible because, based on our pilot trial, the intervention (default options) is expected to alter choices without altering the odds of completing an $\mathrm{AD}$ at all. To show how we will accomplish this, consider the three analyses described in figure 2. First, we will conduct ITT analyses using linear regression, adjusting for centre, ${ }^{53}$ to compare the effects of assignment to complete ADs with different default options on the outcomes of interest. This approach uses data from all randomised participants, and provides the truest test of the overall effectiveness of the intervention (figure 2A). However, the ITT analysis does not provide a specific test of the effects of choices made in ADs, because these effects will be diluted by the fact that many randomised patients will not complete their assigned ADs. Furthermore, among patients who do return completed ADs, not all will stick with their assigned default choice. To surmount this problem, researchers sometimes conduct per-protocol analyses, which, in this case, would compare patients who complete ADs and stay with their assigned defaults in one arm with those who do the same in the other arms (figure 2B). However, perprotocol analyses are likely to be biased by selection effects: patients who complete ADs and choose comfort care are likely different from those who do not complete ADs or make other choices in completed ADs, and these underlying differences may influence outcomes such as quality of life. ${ }^{54}$

To surmount the limitations of ITT analyses in estimating the specific efficacy of choices in ADs, and of perprotocol analyses in providing biased estimates of such efficacy, we will conduct a two-stage least-squares regression in which the randomisation arm is modelled as an $\mathrm{IV}^{55}{ }^{56}$ in CATE analyses. ${ }^{57-60}$ Owing to the randomisation being stratified on the centre, the analysis will also adjust for the centre. ${ }^{53}$ Such analyses, also used in our
Figure 2 Methods of inferring the causal effects of choices made in advance directives (ADs).
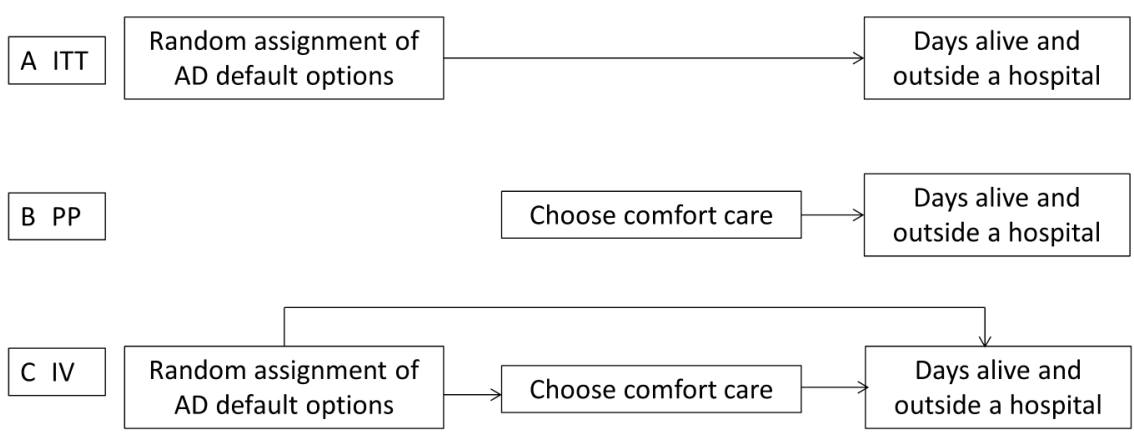
recent randomised trial of behavioural economic interventions for smoking cessation, ${ }^{61}$ use data on all randomised participants to estimate the effects of specifying any treatment choice in ADs regardless of group assignment, and after accounting for the possibility that $A D$ completion rates may differ among the three arms (figure 2C) by using the randomisation arm (ie, initial treatment assignment) as the IV. Thus, the estimated effect of the choices patients make is adjusted for the percentage of assigned patients who complete an $\mathrm{AD}$ at all, and the percentage who opt out from their assigned default option. Unlike a per-protocol analysis, this IV approach uses data on all randomised patients, and then adjusts for $\mathrm{AD}$ completion rates, thereby attenuating the selection effects.

This approach requires the use of principal stratification methods ${ }^{62}$ to formulate the causal quantities of interest and determine the proportions of patients in each arm who would choose comfort care if they were assigned to complete each version of the $\mathrm{AD}$. The analysis assumes that all patients who would choose comfort care in a standard $\mathrm{AD}$ would also choose it in an $\mathrm{AD}$ that defaults to comfort care, and that all patients who would choose comfort care in an $\mathrm{AD}$ that defaults to aggressive care would also choose it in a standard $\mathrm{AD}$ or an AD that defaults to comfort care. Coupled with the possibilities that some participants would never return an $\mathrm{AD}$, and that others would return an $\mathrm{AD}$ but not choose comfort care regardless of group assignment, this creates five compliance classes (principal strata) of participants (table 3). ${ }^{19} 63$

Each patient has three potential outcomes, listed below. Only one of the potential outcomes can be observed, the outcome corresponding to the actual intervention the patient received. For simplicity, we illustrate this with a binary endpoint-whether or not patients would have a high quality of life in the future:

$\mathrm{Y}_{\mathrm{i}}^{\mathrm{A}}=$ whether patient $\mathrm{i}$ would have high quality of life if assigned to complete an aggressive-default $\mathrm{AD}$;

$\mathrm{Y}_{\mathrm{i}}^{\mathrm{S}}=$ whether patient $\mathrm{i}$ would have high quality of life if assigned to complete a standard $\mathrm{AD}$;

Table 3 Compliance classes to estimate the effects of choices made in advance directives $(A D)$

I. Patients would not complete an AD regardless of group assignment

II. Patients would complete an AD but not choose comfort care regardless of group assignment

III. Patients would complete an $A D$ and only choose comfort care if assigned to the comfort-default $A D$

IV. Patients would complete an $A D$ and choose comfort care if assigned to the comfort-default $A D$ or standard $\mathrm{AD}$

V. Patients would complete an $A D$ and choose comfort care regardless of group assignment
$\mathrm{Y}_{\mathrm{i}}^{\mathrm{C}}=$ whether patient $\mathrm{i}$ would have high quality of life if assigned to complete a comfort-default $\mathrm{AD}$.

Our analytic approach will assume the exclusion restriction that $\mathrm{AD}$ assignment only influences the potential outcomes through the causal pathway of determining which type of care the patient chooses through the $\mathrm{AD}{ }^{56}$ However, this assumption is likely to hold in this case, because the randomly assigned IV-which of three versions of an $\mathrm{AD}$ is offered-would not influence outcomes unless it modified the probability of $\mathrm{AD}$ completion or the choices made in the ADs. Further, we will do a sensitivity analysis to examine how violations of the exclusion restriction would influence our results. ${ }^{60}$

In all models, clinic will be entered as a random effect to adjust for potential clustering within clinics and to mitigate confounding by clinic. ${ }^{64}$ Gender, race and diagnosis category will be included in all multivariable models based on prespecified hypotheses, and others will be added if their inclusion-singly or jointly-modifies the coefficient for the randomised exposure by $\geq 15 \%$. $^{65}$ Planned subgroup analyses will be conducted across groups defined by age, race, ethnicity, religion and diagnostic category.

Secondary outcomes will be analysed using logistic, linear or quantile regression, as appropriate. The number of hospital and ICU admissions will be analysed as count data. Costs will be inflated to the date on which analyses are performed using the US gross domestic product deflator. ${ }^{66}$ Hospice usage will be analysed as both the time from $\mathrm{AD}$ completion to hospice enrolment, and the duration of hospice usage prior to death.

\section{Sample size}

We calculate our sample size as that required to rule out a significant reduction in HFDs attributable to random assignment to any AD default. This approach entails non-inferiority tests of data from a Poisson distribution, such that we seek to reject the hypothesis of a rate ratio (RR) for HFDs that is significantly $>1.0$. By enrolling 270 patients who complete ADs-90 in each of the three arms-we will obtain at least $80 \%$ power to demonstrate non-inferiority up to a margin of an RR for HFDs $\geq 1.18$ associated with use of one $\mathrm{AD}$ type. This calculation is based on: (1) a one-sided $\alpha$ of 0.05 , yielding an upper confidence limit on the observed RR that falls entirely below an RR of 1.18; (2) a mean number of HFDs in the control group of 100 , such that a RR of 1.18 would correspond to $15(15 \%)$ fewer HFDs in a given $\mathrm{AD}$ group (100/85=1.18); (3) an allowance for considerable dispersion in the distribution of HFDs; (4) no loss to follow-up because all deaths and hospitalisations will be checked against the Social Security Death Index and Pennsylvania Health Care Cost Containment Council (PHC4), respectively; (5) an allowance for two primary hypotheses tests (comparing both the comfort-default and life-extension default arms to the control arm) and (6) a true RR of 1.0. This final choice reflects our hypothesis that assignment to all three ADs will produce 
equivalent numbers of HFDs. If the true RR is below 1.0 (eg, the comfort default increases HFDs), power would increase considerably. Further, because simulations used to generate these sample size estimates included scenarios with extreme assumptions of data dispersion, and the proposed sample sizes incorporate this conservative assumption, our observed power is likely to be higher than stated.

\section{ETHICS AND DISSEMINATION Ethics}

This study has been approved by the University of Pennsylvania IRB (protocol \#819325) and the IRB at the University of Pittsburgh (PRO14020311). This study is guided by both a Data Safety and Monitoring Board (DSMB) and an External Ethics Advisory Board. The DSMB consists of three individuals with expertise in human subjects research, vulnerable populations, bioethics, clinical trials, decision-making, palliative care and biostatistics. Specifically, the DSMB includes the Chair of Vulnerable Subjects Research at the National Institute of Health, the Chair of Palliative Care at Massachusetts General Hospital, and a statistician at Stanford University. The DSMB reviewed and approved the research protocol and plans for data and safety prior to the start of recruitment. Additionally, DSMB members are evaluating the progress of the trial and making recommendations to ensure that any and all issues are addressed, including decisions about the termination of individual study arms or the study itself. The External Advisory Board is comprised of four noted scholars in health law, palliative care and research ethics; the board meets, as needed, to advise the investigators on any unforeseen challenges related to the ethical conduct of the trial.

The potential risks to human subjects in this project include: (1) risks of breach of confidentiality of personal health information; (2) risks of emotional distress from being asked to contemplate or discuss end-of-life care and (3) potential untoward impacts on patients or family members, including changes in quality of life, duration of life, satisfaction with end-of-life care, surrogate bereavement perceptions of the quality of dying or an undesired change in intervention usage at the end of life. To minimise these risks, our study employs numerous safeguards to protect human subjects. These include an experienced and well-trained study team, a robust informed consent process, state-of-the-art data security, a DSMB and an External Ethics Advisory Board consisting of some of the leading experts in the field.

\section{Dissemination}

In addition to presentation at scientific meetings and publication in scholarly journals, we plan to leverage resources at Penn and Pitt to place our results in the public domain where they can be openly discussed before any policy changes are recommended. This includes developing and implementing strategies to describe results in ways that key stakeholders can understand and implement.

\section{DISCUSSION}

Although most seriously ill Americans wish to avoid burdensome and invasive therapies at the end of life, aggressive care is provided by default - that is, unless otherwise requested. This randomised trial seeks to confirm our prior findings that using different default options in ADs affects patients' stated treatment goals and preferences. In addition, the current trial seeks to leverage presumed changes in these treatment goals and preferences to assess the impact of such choices on a series of clinical, economic and patient-reported and surrogate-reported outcomes. Ultimately, we aim to establish whether a simple and readily scalable intervention-changing default options in ADs-can meaningfully improve patients' quality of life and reduce resource usage without reducing the number of days that patients are alive and living outside of an acute-care hospital.

\section{TRIAL STATUS}

At the time of manuscript submission, 288 patients from the University of Pennsylvania clinics and 168 patients from clinics at the University of Pittsburgh have consented to participate and been randomised, and 208 have returned ADs and been debriefed.

\section{Author affiliations}

${ }^{1}$ Fostering Improvement in End-of-Life Decision Science (FIELDS) Program, Perelman School of Medicine, University of Pennsylvania, Philadelphia, Pennsylvania, USA

${ }^{2}$ Center for Clinical Epidemiology and Biostatistics, Perelman School of Medicine, University of Pennsylvania, Philadelphia, Pennsylvania, USA

${ }^{3}$ Department of Statistics, The Wharton School, University of Pennsylvania, Philadelphia, Pennsylvania, USA

${ }^{4}$ University of Pittsburgh School of Medicine, Pittsburgh, Pennsylvania, USA

${ }^{5}$ Center for Behavioral Decision Research, Carnegie Mellon University, Pittsburgh, Pennsylvania, USA

${ }^{6}$ Department of Medicine, Perelman School of Medicine, University of Pennsylvania, Philadelphia, Pennsylvania, USA

${ }^{7}$ Center for Health Incentives and Behavioral Economics at the Leonard Davis Institute of Health Economics, Perelman School of Medicine, University of

Pennsylvania, Philadelphia, Pennsylvania, USA

${ }^{8}$ Department of Medical Ethics and Health Policy, Perelman School of Medicine, University of Pennsylvania, Philadelphia, Pennsylvania, USA

Contributors NBG, EC, DSS, ABT, RMA, DBW, DCA, GL, KGV, CLB and SDH contributed to the study design and protocol. NBG, EC and SDH drafted the protocol manuscript. DSS, ABT, RMA, DBW, DCA, GL, KGV and CLB provided critical feedback and revisions. All authors have provided final approval of the study protocol.

Funding This work was supported by the Gordon \& Betty Moore Foundation. The funding source has no role in the study design, data collection, analytic plans or manuscript preparation.

Competing interests None declared.

Ethics approval University of Pennsylvania Institutional Review Board and the IRB at the University of Pittsburgh.

Provenance and peer review Not commissioned; externally peer reviewed. 
Open Access This is an Open Access article distributed in accordance with the Creative Commons Attribution Non Commercial (CC BY-NC 4.0) license, which permits others to distribute, remix, adapt, build upon this work noncommercially, and license their derivative works on different terms, provided the original work is properly cited and the use is non-commercial. See: http:// creativecommons.org/licenses/by-nc/4.0/

\section{REFERENCES}

1. Fields MJ, Cassel CK. Approaching death, improving care at the end of life. Washington DC: National Academy Press, 1997.

2. Fried TR, Bradley EH, Towle VR, et al. Understanding the treatment preferences of seriously ill patients. N Engl J Med 2002;346:1061-6.

3. Facts on dying. 2001. https://nts122.chcr.brown.edu/dying/ 2001DATA.HTM

4. Angus DC, Barnato AE, Linde-Zwirble WT, et al., Robert Wood Johnson Foundation ICU End-Of-Life Peer Group. Use of intensive care at the end of life in the United States: an epidemiologic study. Crit Care Med 2004;32:638-43.

5. Kwok AC, Semel ME, Lipsitz SR, et al. The intensity and variation of surgical care at the end of life: a retrospective cohort study. Lancet 2011;378:1408-13.

6. Smith AK, McCarthy E, Weber E, et al. Half of older Americans seen in emergency department in last month of life; most admitted to hospital, and many die there. Health Aff (Millwood) 2012:31:1277-85.

7. Riley GF, Lubitz JD. Long-term trends in Medicare payments in the last year of life. Health Serv Res 2010;45:565-76.

8. Wright AA, Keating NL, Balboni TA, et al. Place of death: correlations with quality of life of patients with cancer and predictors of bereaved caregivers' mental health. J Clin Oncol 2010:28:4457-64.

9. Wright AA, Zhang B, Ray A, et al. Associations between end-of-life discussions, patient mental health, medical care near death, and caregiver bereavement adjustment. JAMA 2008;300:1665-73.

10. Temel JS, Greer JA, Muzikansky A, et al. Early palliative care for patients with metastatic non-small-cell lung cancer. N Engl J Med 2010;363:733-42

11. Bakitas M, Lyons KD, Hegel MT, et al. Effects of a palliative care intervention on clinical outcomes in patients with advanced cancer: the Project ENABLE II randomized controlled trial. JAMA 2009:302:741-9.

12. Connor SR, Pyenson B, Fitch $\mathrm{K}$, et al. Comparing hospice and nonhospice patient survival among patients who die within a three-year window. J Pain Symptom Manage 2007:33:238-46.

13. Bakitas MA, Tosteson TD, Li Z, et al. Early versus delayed initiation of concurrent palliative oncology care: patient outcomes in the ENABLE III randomized controlled trial. J Clin Oncol 2015;33:1438-45

14. Azoulay E, Pochard F, Kentish-Barnes N, et al. Risk of post-traumatic stress symptoms in family members of intensive care unit patients. Am J Respir Crit Care Med 2005;171:987-94.

15. Detering KM, Hancock AD, Reade MC, et al. The impact of advance care planning on end of life care in elderly patients: randomised controlled trial. BMJ 2010;340:c1345.

16. Wendler D, Rid A. Systematic review: the effect on surrogates of making treatment decisions for others. Ann Intern Med 2011; $154: 336-46$.

17. Siegel MD, Hayes E, Vanderwerker LC, et al. Psychiatric illness in the next of kin of patients who die in the intensive care unit. Crit Care Med 2008;36:1722-8.

18. Silveira MJ, Kim SY, Langa KM. Advance directives and outcomes of surrogate decision making before death. N Engl J Med 2010;362:1211-18.

19. Degenholtz HB, Rhee Y, Arnold RM. Brief communication: the relationship between having a living will and dying in place. Ann Intern Med 2004;141:113-17.

20. Nicholas LH, Langa KM, Iwashyna TJ, et al. Regional variation in the association between advance directives and end-of-life Medicare expenditures. JAMA 2011;306:1447-53.

21. Teno JM, Gruneir A, Schwartz Z, et al. Association between advance directives and quality of end-of-life care: a national study. J Am Geriatr Soc 2007;55:189-94.

22. Fagerlin A, Schneider CE. Enough. The failure of the living will. Hastings Cent Rep 2004;34:30-42.

23. Kirschner KL. When written advance directives are not enough. Clin Geriatr Med 2005;21:193-209, x.

24. Perkins HS. Controlling death: the false promise of advance directives. Ann Intern Med 2007;147:51-7.
25. Tonelli MR. Pulling the plug on living wills. A critical analysis of advance directives. Chest 1996:110:816-22.

26. Tulsky JA. Beyond advance directives: importance of communication skills at the end of life. JAMA 2005;294:359-65.

27. Chapman GB, Li M, Colby H, et al. Opting in vs opting out of influenza vaccination. JAMA 2010;304:43-4.

28. Choi JJ, Laibson D, Madrian BC, et al. Defined contribution pensions: plan rules, participant decisions, and the path of least resistance. In: Poterba JM, ed. Tax policy and the economy. Cambridge, MA: MIT Press, 2002:67-113.

29. Horvat LD, Cuerden MS, Kim SJ, et al. Informing the debate: rates of kidney transplantation in nations with presumed consent. Ann Intern Med 2010;153:641-9.

30. Johnson EJ, Goldstein D. Medicine. Do defaults save lives? Science 2003;302:1338-9.

31. Johnson EJ, Hershey JC, Meszaros J, et al. Framing, probability distortions, and insurance decisions. J Risk Uncertain 1993;7:35-51.

32. Madrian BC, Shea DF. The power of suggestion: Intertia in 401(k) participation and savings behavior. Q J Econ 2001;116:1149-87.

33. Halpern SD, Ubel PA, Asch DA. Harnessing the power of default options to improve health care. N Engl J Med 2007;357:1340-4.

34. Loewenstein G, Brennan T, Volpp KG. Asymmetric paternalism to improve health behaviors. JAMA 2007;298:2415-17.

35. Quill CM, Halpern S. Deciphering the appropriateness of defaults: the need for domain-specific evidence. J Med Ethics 2012;38:721-2.

36. Hart J, Halpern SD. Default options in the ICU: widely used but insufficiently understood. Curr Opin Crit Care 2014;20:662-7.

37. Halpern SD, Loewenstein G, Volpp KG, et al. Default options in advance directives influence how patients set goals for end-of-life care. Health Aff (Millwood) 2013;32:408-17.

38. Carstensen LL. The influence of a sense of time on human development. Science 2006;312:1913-15.

39. Carstensen LL, Isaacowitz DM, Charles ST. Taking time seriously. A theory of socioemotional selectivity. Am Psychol 1999;54:165-81.

40. Shea JA, Micco E, Dean LT, et al. Development of a revised Health Care System Distrust scale. J Gen Intern Med 2008;23:727-32.

41. Harris PA, Taylor R, Thielke R, et al. Research electronic data capture (REDCap) - a metadata-driven methodology and workflow process for providing translational research informatics support. $J$ Biomed Inform 2009;42:377-81.

42. Living Will and Healthcare Power of Attorney. 2007. http://www. acms.org/lw/index.html

43. A controlled trial to improve care for seriously ill hospitalized patients. The study to understand prognoses and preferences for outcomes and risks of treatments (SUPPORT). The SUPPORT Principal Investigators. JAMA 1995;274:1591-8.

44. Barnato $\mathrm{AE}$, Farrell $\mathrm{MH}$, Chang CC, et al. Development and validation of hospital 'end-of-life' treatment intensity measures. Med Care 2009;47:1098-105.

45. Miller FG, Gluck JP Jr, Wendler D. Debriefing and accountability in deceptive research. Kennedy Inst Ethics J 2008;18:235-51.

46. Heyland DK, Cook DJ, Rocker GM, et al. The development and validation of a novel questionnaire to measure patient and family satisfaction with end-of-life care: the Canadian Health Care Evaluation Project (CANHELP) Questionnaire. Palliat Med 2010;24:682-95.

47. Heyland DK, Frank C, Tranmer J, et al. Satisfaction with end-of-life care: a longitudinal study of patients and their family caregivers in the last months of life. J Palliat Care 2009;25:245-56.

48. Cohen SR, Mount BM, Bruera E, et al. Validity of the McGill Quality of Life Questionnaire in the palliative care setting: a multi-centre Canadian study demonstrating the importance of the existential domain. Palliat Med 1997;11:3-20.

49. Cohen SR, Mount BM, Strobel MG, et al. The McGill Quality of Life Questionnaire: a measure of quality of life appropriate for people with advanced disease. A preliminary study of validity and acceptability. Palliat Med 1995;9:207-19.

50. O'Connor AM. Validation of a decisional conflict scale. Med Decis Making 1995;15:25-30.

51. Zhang B, Nilsson ME, Prigerson HG. Factors important to patients' quality of life at the end of life. Arch Intern Med 2012;172:1133-42.

52. Horowitz M, Wilner N, Alvarez W. Impact of Event Scale: a measure of subjective stress. Psychosom Med 1979;41:209-18.

53. Kahan BC, Morris TP. Improper analysis of trials randomised using stratified blocks or minimisation. Stat Med 2012;31:328-40.

54. Sommer A, Zeger SL. On estimating efficacy from clinical trials. Stat Med 1991;10:45-52.

55. Newhouse JP, McClellan M. Econometrics in outcomes research: the use of instrumental variables. Annu Rev Public Health 1998;19:17-34 
56. Angrist JD, Imbens GW, Rubin DW. Identification of causal effects using instrumental variables. J Am Stat Assoc 1996;91:444-55.

57. Cheng J, Small D. Bounds on causal effects in three-arm trials with noncompliance. J R Stat Soc 2006;68: 815-36.

58. Cheng J, Small D, Tan Z, et al. Efficient nonparametric estimation of causal effcts in randomized trials with noncompliance. Biometrika 2009;96:19-36.

59. Sussman JB, Hayward RA. An IV for the RCT: using instrumental variables to adjust for treatment contamination in randomised controlled trials. BMJ 2010;340:c2073.

60. Baiocchi M, Cheng J, Small DS. Instrumental variable methods for causal inference. Stat Med 2014;33:2297-340.
61. Halpern SD, French B, Small DS, et al. Randomized trial of four financial-incentive programs for smoking cessation. N Engl J Med 2015;372:2108-17.

62. Frangakis $\mathrm{CE}$, Rubin DB. Principal stratification in causal inference. Biometrics 2002;58:21-9.

63. Sudore RL, Fried TR. Redefining the 'planning' in advance care planning: preparing for end-of-life decision making. Ann Intern Med 2010;153:256-61.

64. Localio AR, Berlin JA, Ten Have TR, et al. Adjustments for center in multicenter studies: an overview. Ann Intern Med 2001;135:112-23.

65. Hosmer DW, Lemeshow S, Sturdivant RX. Applied logistic regression. 2nd edn. John Wiley \& Sons, 2000.

66. U.S. Department of Commerce Bureau of Economic Analysis. http:// www.bea.gov/national/ (accessed 16 Apr 2015). 